\title{
The use of a Multilayer Perceptron for detecting new human settlements from a time series of MODIS images
}

\author{
B.P. Salmon ${ }^{\mathrm{a}, *}$, J.C. Olivier ${ }^{\mathrm{a}}$, W. Kleynhans ${ }^{\mathrm{a}}$, K.J. Wessels ${ }^{\mathrm{b}}$, F. van den \\ Bergh $^{b}$, K.C. Steenkamp ${ }^{b}$ \\ ${ }^{a}$ Department of Electrical, Electronic and Computer Engineering, University of Pretoria, \\ Lynnwood Road, Hillcrest, Pretoria 0002, South Africa \\ ${ }^{b}$ Remote Sensing Research Unit, Meraka Institute, Council for Scientific and Industrial \\ Research, Meiring Naude Road, Brummeria, Pretoria 0002, South Africa
}

\begin{abstract}
This paper presents a novel land cover change detection method that employs a sliding window over hyper-temporal multi-spectral images acquired from the 7 bands of the MODerate-resolution Imaging Spectroradiometer (MODIS) land surface reflectance product. The method uses a Feedforward Multilayer Perceptron (MLP) for supervised change detection that operates on multi-spectral time series extracted with a sliding window from the dataset. The method was evaluated on both real and simulated land cover change examples. The simulated land cover change comprises of concatenated time series that are produced by blending actual time series of pixels from human settlements to those from adjacent areas covered by natural vegetation. The method employs an iteratively retrained MLP to capture all local patterns and to compensate for the time-varying climate change in the geographical area. The iteratively retrained MLP was compared to a classical batch mode trained MLP. Depending on the length of the temporal sliding window used, an overall change detection accuracy between $83 \%$ and $90 \%$ was achieved. It is shown that a sliding window of 6 months using all 7 bands of MODIS data is sufficient to detect land cover change reliably. Window sizes of 18 months and longer provide minor improvements to classification accuracy and change detection performance at the cost of longer time delays.
\end{abstract}

Keywords: Change detection, classification, feedforward neural networks,

\footnotetext{
*Corresponding Author. Tel.: +27 12841 3207, Fax: +27 128413124

Email address: bsalmon@csir.co.za (B.P. Salmon)
} 
satellite, time series.

\section{Introduction}

Land cover change detection at regional or global scales, using hypertemporal, multi-spectral coarse resolution satellite data, has been a highly desired goal of the environmental remote sensing community (Townshend and Justice, 1988; Hansen and DeFries, 2004). Land cover change often indicates land use change with major socio-economic impacts, while the transformation of vegetation cover (e.g. deforestation, agricultural expansion, urbanization) has significant impacts on hydrology, ecosystems and climate (DeFries et al., 2002; Foley et al., 2005). Digital change detection encompasses the quantification of temporal phenomena from multi-date imagery that is most commonly acquired by satellite-based multi-spectral sensors (Lunetta et al., 2006).

Due to the complexity and non-parametric nature of land cover classification and change detection, machine learning methods are widely regarded as the most viable option for classification and change detection (DeFries and Chan, 2000; Lu and Weng, 2007). Supervised ARTMAP neural networks have been used for global land cover classification (Gopal et al., 1999), and forest characterization (Carpenter et al., 1999). Other neural networks have been used to detect change in pairs of SPOT images (Nemmour and Chibani, 2005), 5 years of MODIS data (Westra and Wulf, 2007) or a combination of MODIS, Multiangle Imaging SpectroRadiometer (MISR) data and high resolution imagery (Braswell et al., 2003).

The above-mentioned examples and many other change detection studies rely either on image differencing, post-classification comparison methods or change trajectory analysis (for reviews see Lu et al. (2004)). None of these methods fully capitalize on the high temporal sample rate of coarse resolution satellite data. Daily and multi-day composite data provided by coarse resolution satellite images allow the observation of seasonal land surface reflectance patterns which facilitates distinguishing different land cover types and provides a signal for applying advanced time series analysis (Loveland et al., 1995).

A temporal sliding window is regularly used in variety of fields to extract streaming values from an individual time series (Vermaak and Botha, 1998; Wang et al., 2005). It was shown that by employing correct feature extraction 
techniques on the temporal sliding window (Salmon et al., 2011), the analysis of the time series is not hindered by the limitations inherent in most studies (Keogh and Lin, 2005). Land cover change is defined here as the transition in class label of a pixel's time series from one class to another class, after which it remains in the newly assigned class for the remainder of the time series.

The case for using a Multilayer Perceptron (MLP) for land use classification rather than maximum likelihood (ML) methods was made by Paola \& Schowengerdt (Paola and Schowengerdt, 1995), where it was shown that a MLP appeared to learn the complex non-linear interdependencies of the multidimensional time series data derived from multiple spectral bands.

The MODIS global land cover product uses a supervised approach to derive estimates of class conditional probabilities for each class at each pixel (Friedl et al., 2010). The objective of this paper is to introduce the concept of supervised MLP operating on a temporal sliding window and evaluate its performance on detecting both real and simulated land cover change, on a per pixel basis, using the Normalized Difference Vegetation Index (NDVI), the first two spectral bands and all seven spectral bands of the time series extracted from the MODIS land surface reflectance product.

The paper is organized as follows. Section 2 presents the methodology used, while section 3 presents our experimental setups. Section 4 presents the results for the change detection on land cover change. Section 5 presents the conclusions.

\section{Methodology}

\subsection{Study Areas}

The Limpopo Province in northern South Africa is largely covered by natural vegetation, while the expansion of human settlements (often informal and unplanned) is the most pervasive form of new land cover change in South Africa. Study areas were selected where settlements and natural vegetation occur in close proximity to ensure that the rainfall, soil type and local climate were similar over both land cover types. The study areas were: 1) Koelemansrus area with a total area of $195.5 \mathrm{~km}^{2}$ located $17 \mathrm{~km}$ south-west of Mokopane (28.85E, 24.23S), 2) Seshego area with a total area of 123.5 $\mathrm{km}^{2}$ located $10 \mathrm{~km}$ north-west of Polokwane (29.40E, 23.81S), and 3) Sekuruwe area with a total area of $124.0 \mathrm{~km}^{2}$ located $28 \mathrm{~km}$ north of Mokopane (28.94E, 23.94S). 


\subsection{MODIS time series data}

The MODIS (MCD43A4, Collection V005) 500-meter, Nadir and Bidirectional Reflectance Distribution Function (BRDF) adjusted spectral reflectance bands were used, as it significantly reduces the anisotropic scattering effects of surfaces under different illumination and observation conditions (Wanner et al., 1997; Schaaf et al., 2002). The data set provides a sample on a rolling 8 day interval based on 16 days of MODIS surface reflectance composite period, for each of the seven spectral bands at 500 meter resolution. The 500 meter resolution spectral bands were considered to illustrate the advantages of using additional spectral bands in the analysis, since the other spectral bands are only available at 500 meter resolution and BRDF corrected. For each pixel in each study area a time series was extracted for all 7 bands from the data set (tile H20V11) (year 2000-2008). South Africa has low cloud obscuration (Roy et al., 2005) and the quality flags were used to identify the small number of low quality samples and replaced them with interpolants obtained using a cubic spline fitted through temporal neighbours.

\section{Place Table 1 here}

\subsubsection{Training data sets}

A supervised MLP was used for change detection (see Section 2.4) and thus the classifier required training data. A training set must be defined for each study area to ensure that the classifier captures local patterns. Clusters of close adjacent pixels were selected for the training area, using manual visual interpretation of SPOT2 (2000) and SPOT5 (2006) high resolution images to ensure that none of the time series experienced any land cover change during the study period. The number of time series selected for each class, per study area, is given in Table 1 . These training sets represent the two valid classes for the MLP in training mode.

\subsubsection{Validation data set}

The MLP relies on independent validation data sets (not included in training phase) to verify the MLP's generalization (Bishop, 1996). These data sets were generated using the procedure followed for the training set by using the same SPOT2 (2000) and SPOT5 (2006) high resolution imagery. Table 1 represents the number of time series associated with each class in both study areas. 


\subsubsection{Testing data sets: Real and simulated land cover change}

\section{Place Figure 1 here}

Real land cover change was identified in the Sekuruwe study area. This land cover change amounted to $29.25 \mathrm{~km}^{2}$ of land cover conversion from natural vegetation to human settlement. The land cover conversion was verified by manual visual interpretation of high resolution SPOT2 (2000) and SPOT5 (2006) imagery in the Sekuruwe study area (Fig. 1). The land cover change detection algorithm presented within this paper was tested on these land cover change instances and the results are presented in section 4 .

Even though human settlement expansion is one of the most pervasive forms of new land cover change in South Africa, information on this form of land cover change is poorly documented, and vital details such as the date of land cover conversion cannot be determined reliably. Thus to study the behaviour of the algorithm with respect to change detection delay (a vital statistic), the real change data set was augmented with a simulated land cover change data set where the date of change is explicitly known and thus it becomes possible to quantitatively evaluate the change detection method. The simulation of land cover change in addition to the real change time series made it possible to control both the type, rate and timing of the land cover change. These are a function of both anthropogenic activities and environmental conditions and could be incorporated within a model where these parameters were known.

The simulated land cover change time series set was created by concatenating a set of actual time series from natural vegetation class (settlement class) to another set of actual time series from settlement class (natural vegetation class). As a control, testing sets containing no land cover change were also created by concatenating a set of time series from a natural vegetation class (settlement class) to other set of time series also from a natural vegetation class (settlement class). Hence there were four testing data subsets based on concatenating time series of different combination of time series:

- subset 1: natural vegetation time series (class 1) concatenated to settlement time series (class 2);

- subset 2: settlement time series (class 2) concatenated to natural vegetation time series (class 1); 
- subset 3: settlement time series (class 2) concatenated to another settlement time series (class 2);

- subset 4: natural vegetation time series (class 1) concatenated to another natural vegetation time series (class 1 ).

These four subsets were used to produce a confusion matrix to test if the supervised MLP can detect change reliably on subsets 1 and 2, while not falsely detecting change for subsets 3 and 4 . The number of simulated land cover change time series available for the analysis process was limited by the smallest number of pixels available per class in the three study areas, i.e. there were 248 settlement pixels available in the first study area (Table 1 ), where a third was used for simulated land cover change (84 settlement pixels), a third for training of the MLP (82 settlement pixels), and a third for independent validation (82 settlement pixels).

The concatenation process produces an abrupt change in the time series, which does not necessarily represent the reality of human-induced change such as settlement expansion, that may take several months to develop. The notion of a blending period (linear blend over 12 and 24 months) from one land cover time series to another was initially considered. The blending model does not infer all forms of actual land cover change but did illustrate that the blending period does not affect the ability to correctly detect change but merely delays the date on which the MLP's output classify the correct class (see section 4.3).

\section{Place Figure 2 here}

Effective change detection time $\Delta_{\tau}$ is another parameter that can be evaluated on the simulated land cover change and is defined as the time duration that the classifier is unable to make a correct classification after the sliding window encounters the first acquisition where change is introduced (Figure 2).

\section{Place Figure 3 here}

\subsection{Temporal sliding window and change detection}

The MLP's function is to process time series data and detect land cover change as a function of time. A complete system diagram is provided in Fig. 
3. This required a temporal sliding window that operates on a subsequence of the time series for several combinations of spectral bands (Fig. 4).

\section{Place Figure 4 here}

A subsequence $x_{m p}(t)$ for a given time series $x_{m}(t)$ of length $P_{\max }$, for any specific spectral band, is given as

$$
x_{m p}(t)=\left[x_{m}\left(t_{p}\right) x_{m}\left(t_{p+1}\right) \ldots x_{m}\left(t_{p+Q}\right)\right],
$$

for all the time increments $1 \leq p \leq P_{\max }-Q+1$, where $Q$ is the length of the sliding window and $m, m \in[1, N]$, denotes the spectral band of the MODIS instrument. The sliding window has a length $Q$ of 6,12 , and, 18 months which denotes 23, 46, and, 69 MODIS acquisitions respectively at a fixed rate of 8 day increments within a 16 day composite period. The position of the sliding window $p$ was incremented sequentially by one sample through the time series. Since the delay in change detection in the initial training period was determined by the length of the sliding window $Q$, the effect of its length on the accuracy of change detection was studied.

The fixed acquisition rate of the MODIS product and the seasonality of the vegetation in the study area makes for an annual periodic signal $x_{m}(t)$ that has a phase offset that is correlated with rainfall seasonality and land surface phenology (Kleynhans et al., 2010). The Fast Fourier Transform (FFT) (Oppenheim et al., 1999), computed over the subsequence of length $Q$, produces the frequency spectrum. This decomposes the time sequence's values into components of different frequencies with phase offsets. Computing the magnitude of all the FFT components removes the phase offset, which makes it possible to compensate for the effects of rainfall seasonality (Salmon et al., 2011). This would translate into frequency components in the frequency spectrum that have fixed positions, because of the annual periodic properties of the time series $x_{m}(t)$. The features $T_{m p}(f)$ were extracted from the sliding window $x_{m p}(t)$ by the methodology discussed above as

$$
T_{m p}(f)=\left|\mathcal{F}\left(x_{m p}(t)\right)\right|,
$$

where $\mathcal{F}(\cdot)$ is the Fourier transform. The seasonal attribute typically associated with MODIS time series and the slow temporal variation relative to the acquisition interval (Lunetta et al., 2004) made certain lower frequencies dominant. This reduces the number of features used to represent the feature 
space and thus reduces the dimensionality. These dominant components were extracted from the $N$ spectral bands to represent the feature vector inputs to the MLP (Fig. 3). These dominant components were extracted as

$$
X_{m p}=\left[T_{m p}\left(f_{1}\right) T_{m p}\left(f_{2}\right) \ldots T_{m p}\left(f_{K}\right)\right] .
$$

Here $m, m \in[1, N]$, denotes the spectral band, and $p, p \in\left[1, P_{\max }-Q+1\right]$, the time increment of the sliding window.

\subsection{Multilayer Perceptron}

The MLP comprises an input layer, one hidden layer and an output layer. All hidden and output nodes used a tangent sigmoid activation function in each node. The input layer accepts input vectors for classification, while the output layer represents the likelihood that an input belongs to a specific class every 8 days.

The MLP output was in the range $(-1 ; 1)$, where 1 represents a $100 \%$ certainty of class membership to class 1 (natural vegetation) given the input vector, while a -1 represents a $100 \%$ certainty of class 2 (settlement). Good preliminary results were obtained when a temporal moving average filter of length 3 (24 days) was applied to the output class membership stream of the supervised MLP. The moving average window smoothed out the transitory oscillations in class labels caused by higher uncertainty in the classification. For correct change detection it was required that the MLP correctly classified both time series according to a threshold $T_{h}$ before and after the change was introduced. This threshold was used to impose a strict evaluation on the output class membership stream to ensure that coherent classification was achieved before land cover change is declared. This means that the MLP's tangent sigmoid activation function output can be classified as vegetation in the range $\left[T_{h}, 1\right]$, settlement in the range $\left[-1,-T_{h}\right]$, and uncertain in the range $\left(-T_{h}, T_{h}\right)$.

The weights in the training phase of the MLP were determined using a steepest descent gradient optimization method, with gradients estimated using backpropagation (Bishop, 1996). The validation set was used for initial MLP architecture optimization by testing the generalization error to identify overfitting of the network for each study area. The MLP architecture was optimized each time for sliding window length $Q$, number of spectral bands and training mode (batch mode and iteratively retraining mode). 
In the batch mode (Bishop, 1996) all the incremental, sliding windows between the year 2000 and the year 2008 were used as initial training inputs to the MLP. The experiments were conducted for the 8 years without any retraining. The iteratively retrained MLP is proposed to compensate for the inter-annual variability between years due to the rainfall variability. The iteratively retrained MLP was trained to recognize data from the training set within the sliding window at time $p$ in the time series, and was used to classify the data from the testing set within the sliding window at time $p$. This retraining at each time increment caused a small adaptation of the weights, and has low complexity due to the small incremental MLP weight changes over each 8 day increment. These small MLP weight changes only required 300 epochs at each time increment for network adaptation.

\section{Experimental Setup}

For evaluation, the sliding window length was set to 6,12 and 18 months in all experiments presented in section 4 . It was found that the first few components dominated the frequency spectrum (Lhermitte et al., 2008) because of the seasonal attribute typically associated with MODIS time series and the slow temporal variation relative to the acquisition interval (Lunetta et al., 2004). This reduced the number of features used to represent the feature space and thus reduced the dimensionality, making the MLP a feasible option. The number of components that were extracted in (3), was determined over all three input vectors. Starting with only one component, additional components were added to each input vector incrementally until no further meaningful performance improvement was observed. For this reason the first five components were extracted and used as information on the land cover type in all experiments. The number of nodes in each layer is expressed in table 9 .

Three different input vectors utilising different spectral bands were evaluated. The input vectors were used as the input to the MLP (Fig. 3) and is given as

$$
X_{p}(f)=\left[X_{1 p}(f) X_{2 p}(f) \ldots X_{N p}(f)\right] .
$$

Here $N$ denotes the number of spectral bands, and $p, p \in\left[1, P_{\max }-Q+1\right]$, the time index of the sliding window. The first input vector was the NDVI time series $(N=1)$. This is where the NDVI was computed for $x_{m p}(t)$ in (1), 
which uses a combination of the first two spectral bands of the MODIS instrument. The second input vector was to use the first two spectral bands separately $(N=2)$. The last input vector used all seven spectral bands separately $(N=7)$. The three different input vectors were evaluated in all experiments. The decision threshold $T_{h}$ introduced in section 2.4 was set to 0.1 in all the experiments. This value was fixed to accommodate all the experiments and to ensure fairness.

These experiments were conducted in all three study areas introduced in section 2.1. The number of time series are tabulated in Table 1. These study areas include land cover validation time series that undergo no change and simulated land cover change. Real land cover change was available in the Sekuruwe study area.

The training pixels (section 2.2.1) were used in two different modes to train the MLP described in section 2.4, namely batch mode and iteratively retrained mode. Both modes were evaluated in all the experiments.

In the evaluation of each study area, the classification accuracy on the validation pixels (section 2.2.2) is reported in section 4.1. The land cover change detection accuracies were measured on the simulated land cover change time series (section 2.2.3) and are reported in section 4.2. The land cover change detection accuracies were also measured on real land cover change time series (section 2.2.3) that were present in the Sekuruwe study area and reported in section 4.4. The effective change detection time $\Delta_{\tau}$ was calculated on the augmented simulated land cover change, since the time of land cover change can be controlled within the time series, and is reported in section 4.5.

The notion of a blending period that was introduced in section 2.2.3 is illustrated in section 4.3. The blending period was only tested on the simulated land cover change time series since the rate of land cover change could be controlled.

\section{Results and Discussion}

Place Table 2 here

Place Table 3 here 


\subsection{Classification accuracy - No land cover change}

In the batch mode trained MLP, relatively low settlement classification accuracy and high coefficient of variation was observed using the NDVI and two spectral bands' time series (Table 2). In the case of the iteratively retrained mode MLP, similar classification accuracies were observed when all seven spectral bands were used when compared to the batch mode trained MLP, unlike the general improvement that was observed with the other inputs vectors. Overall, classifiers using all seven spectral bands as input performed better than the other experiments that were conducted. The seven band input vector improved the average classification accuracies significantly compared to the other input vectors for the batch mode trained MLP (Table 2) and for the iteratively retrained MLP (Table 3). The coefficient of variation decreased relative to the other input vectors when using the seven band input vector for the batch mode trained MLP (Table 2) and the iteratively retrained MLP (Table 3). An improvement was observed in all the experiments when longer sliding windows (12, 18 months) were used.

Place Table 4 here

Place Table 5 here

\subsection{Change detection accuracy - Simulated land cover change}

The low classification accuracies and high coefficient of variation reported in section 4.1 were reflected in the low change detection accuracies on the simulated land cover change. In the batch mode trained MLP, a relative good change detection accuracy was observed for the Koelemansrus and Seshego study area when a sliding window length of 12 months and longer was used. The seven spectral bands produced the highest land cover change detection accuracies in all the study areas.

In the case of the iteratively retrained mode MLP, improvements were observed in the majority of the experiments when compared to the batch mode trained MLP. Similar land cover change detection accuracies were observed for the seven band input vector, but a significant improvement (up to $71 \%$ ) was observed with the NDVI time series and the two spectral bands time series.

The conclusion is that the seven spectral bands using the iteratively retrained mode MLP performed the best for detecting land cover change on 
the simulated land cover change time series. A general improvement was observed in all experiments when the sliding window length was increased to 12 months and longer.

\section{Place Figure 5 here}

\subsection{Effect of blending period - Simulated land cover change}

Fig. 5 shows the classification output of the MLP after the time series of the surface reflectance data of a natural vegetation time series was blended with that of a settlement time series over a 12 and 24 month period versus abrupt concatenation. The MLP created transitory oscillations in the output labels during the interval that the land cover type was changing. The introduction of a blending period did not affect the ability to correctly detect land cover change, but merely delayed the period before the MLP classifies into the new class.

\section{Place Table 6 here}

\subsection{Change detection accuracy - Real land cover change}

The Sekuruwe study area was the only study area that presented real land cover change that could be evaluated. For both the batch mode trained MLP and the iteratively retrained MLP the real land cover change detection accuracies were similar to that of the simulated land cover change detection accuracies presented in section 4.2. Just like in the simulated land cover change experiments of section 4.2, a longer sliding window length yielded better performance. The land cover change detection accuracies improved when the sliding window length $Q$ was increased.

\section{Place Table 7 here}

\section{Place Table 8 here}

\subsection{Effective change detection time - Simulated land cover change}

Preliminary experiments showed that a shorter sliding window had a shorter effective change detection time $\Delta_{\tau}$, which meant the MLP detected land cover change faster since the data inside the temporal sliding window was closer in time to the land cover change event. The results on the effective change detection time $\Delta_{\tau}$ for the simulated land cover change sets for sliding 
window sizes of 6,12 and 18 months using the NDVI, two spectral bands, and all seven spectral bands operating on both the batch mode trained MLP and the iteratively retrained mode MLP are provided in Table 7 and Table 8. Each entry in both tables gives the effective change detection time in days for each of the study areas, calculated over 15 repeated independent experiments.

In batch mode trained MLP, the disadvantage was that the average classification accuracy and land cover change detection accuracy was relatively poor when the sliding window length $Q$ was shorter than 12 months. The NDVI time series were more prone to incorrect classifications during the change process than the two and seven spectral band time series approaches, i.e. the effective change detection time $\Delta_{\tau}$ could not be calculated in the Koelemansrus study area with a sliding window of 6 months due to constant oscillations in the MLP's output stream. A relative small improvement trend was observed in the effective change detection time when the sliding window length was shortened.

In the case of the iteratively retrained mode MLP, improvements were observed over all the experiments when compared to the batch mode trained MLP. The lowest relative effective change detection time was observed when the seven spectral band input vector was used in the MLP.

\section{Place Table 9 here}

\subsection{Computational complexity}

Table 9 shows the computational times for both the batch mode MLP and iteratively retrained MLP computed in the Sekuruwe study area. A Dell PowerEdge 1955 blade, Intel Xeon 5355 (Quad-Core) 2.66GHz, 8GB RAM system was used to compute the computational times for the different combinations. The fastest computational time is used as a base comparison for the other experimental computational times. The other computation times are expressed as fractional increases of this base. The fastest computational time was the iteratively retrained MLP operating using a sliding window length $Q$ of 6 months and the NDVI time series as the input vector $X_{p}(f)$. An increase of less than $25 \%$ was observed in the computational times when other combinations of NDVI or the 2 spectral bands were used as input vector $X_{p}(f)$. An increase of more than $65 \%$ was observed in the computational time when 7 spectral bands was used as input vector. 


\section{Conclusions}

This paper demonstrated that a MLP operating on a temporal sliding window of MODIS time series data was able to detect land cover change after initial training. Like most other supervised machine learning methods, the method proposed here was (i) dependent on periodic high resolution imagery for redefining the training sets over time and (ii) required to be robust to land cover changes and other errors occurring within these training sets (DeFries and Chan, 2000).

From all the results presented in section 4 it was clear that better change detection can be ensured when all seven bands of the MODIS sensor were used, producing higher classification accuracies and reducing the effective change detection time $\Delta_{\tau}$. The iteratively retrained MLP using all seven spectral bands performed the best out of all the experiments conducted in all the study areas. The iteratively retrained mode MLP accurately detected simulated land cover change at accuracy rates of $89 \%-94 \%$ (Table 5) and $83 \%-90 \%$ (Table 6) for real land cover detection in the Sekuruwe study area, and has a lower effective change detection time than the batch mode trained MLP.

The iteratively retrained MLP performed better than the batch mode MLP (Table 2 versus Table 3 ) because the iteratively retrained MLP had the advantage of learning the most recent spectral properties of land cover types, as time progresses. The iteratively retrained MLP takes cognisance of what is within the temporal sliding window to compensate for short-term inter-annual climate variability and adapts to longer-term trends in climate without confusing any of these with land cover change, which has often been a problem with other regional land cover change studies (Vanacker et al., 2005; Olsson et al., 2005).

The temporal sliding window approach can also be applied in combination with a variety of other classifiers or machine learning methods. The methods introduced here will next be applied to multiple land cover classes and an entire MODIS tile.

The main trade off in this study was between the effective change detection time $\Delta_{\tau}$ and the length of the temporal sliding window $Q$. Higher classification accuracy and land cover change detection rates can be accomplished when the temporal sliding window length $Q$ is increased from 6 months to 18 months (Table 3 and Table 5), but at the loss of increasing the effective change detection time $\Delta_{\tau}$ (Table 7 and Table 8). The loss in classification 
accuracy is less important when related to the reduction in length of the temporal sliding window length $Q$ (Table 3 ), when compared to the excessive increase in effective change detection time $\Delta_{\tau}$ when the temporal sliding window length $Q$ was extended (Table 5).

Land cover change can be detected by either using the NDVI time series or the first two spectral bands' time series of the MODIS data (Friedl et al., 2010), but in this paper the land cover classification (Table 3) and change detection (Table 5) was found to be more reliable and faster (Table 8) when the iteratively retrained MLP was used with all seven spectral bands.

The main operational challenge will be the generation of training data from the ancillary land cover data which is representative of the environmental diversity and diverse land uses contained within such an extensive area. The method proposed is a contribution to the advancement of land cover change detection using a machine learning approach on satellite time series data. In the foreseeable future change detection systems based on coarse resolution satellites could ultimately be used to alert and task high resolution satellites to acquire images for an area of interest within an autonomous Earth Observation sensorweb (Chien et al., 2005).

\section{Acknowledgements}

The research was funded by the CSIR Strategic Research Panel. Alex Fortesque and Naledzani Mudau of CSIR, Satellite Application Centre (SAC) provided data on settlements.

\section{References}

Bishop, C., 1996. Neural networks for pattern recognition, 1st Edition. Oxford University Press, New York, USA.

Braswell, B. H., Hagen, S. C., Frolking, S. E., Salas, W. A., October 2003. A multivariable approach for mapping sub-pixel land cover distributions using MISR and MODIS: application in the Brazilian Amazon region. Remote Sensing of Environment 87 (2-3), 243-256.

Carpenter, G. A., et al., December 1999. A neural network method for efficient vegetation mapping. Remote Sensing of Environment 70 (3), 326-338.

Chien, S., et al., May 2005. An autonomous Earth-Observing Sensorweb. IEEE Intelligent Systems 20 (3), 16-24. 
DeFries, R. S., Bounoua, L., Collatz, G. J., May 2002. Human modification of the landscape and surface climate in the next fifty years. Global Change Biology 8 (5), 438-458.

DeFries, R. S., Chan, J. C. W., December 2000. Multiple criteria for evaluating machine learning algorithms for land cover classification from satellite data. Remote Sensing of Environment 74 (3), 503-515.

Foley, J. A., et al., July 2005. Global consequences of land use. Science 309 (5734), 570-574.

Friedl, M., et al., January 2010. MODIS collection 5 global land cover: Algorithm refinement and characterization of new datasets. Remote Sensing of Environment 114 (1), 168-182.

Gopal, S., Woodcock, C. E., Strahler, A. H., 1999. Fuzzy neural network classification of global land cover from a 1 degrees AVHRR data set. Remote Sensing of Environment 67, 230-243.

Hansen, M. C., DeFries, R. S., November 2004. Detecting long-term global forest change using continuous fields of tree-cover maps from 8-km Advanced Very High Resolution Radiometer (AVHRR) data for the years 1982-99. Ecosystems 7 (7), 695-716.

Keogh, E., Lin, J., August 2005. Clustering of time-series subsequences is meaningless: Implications for previous and future research. Knowledge Information Systems 8 (2), 154-177.

Kleynhans, W., et al., April 2010. Improving land cover class separation using an Extended Kalman Filter on MODIS NDVI time-series data. IEEE Geoscience and Remote Sensing Letters 7 (2), 381-385.

Lhermitte, S., et al., February 2008. Hierarchical image segmentation based on similarity of NDVI time series. Remote Sensing of Environment 112 (2), 506-521.

Loveland, T., et al., June 1995. Seasonal land-cover regions of the United States. Annals of the Association of American Geographers 85 (2), 339355. 
Lu, D., Mausel, P., Brondizio, E., Moran, E., June 2004. Change detection techniques. International Journal of Remote Sensing 25 (12), 2365-2407.

Lu, D., Weng, Q., January 2007. A survey of image classification methods and techniques for improving classification performance. International Journal of Remote Sensing 28 (5), 823-870.

Lunetta, R. S., Johnson, D., Lyon, J., Crotwell, J., February 2004. Impacts of imagery temporal frequency on land-cover change detection monitoring. Remote Sensing of Environment 89 (4), 444-454.

Lunetta, R. S., et al., November 2006. Land-cover change detection using multi-temporal MODIS NDVI data. Remote Sensing of Environment 105 (2), 142-154.

Nemmour, H., Chibani, Y., January 2005. Neural network combination by fuzzy integral for robust change detection in remotely sensed imagery. EURASIP Journal on Applied Signal Processing 2005, 2187-2195.

Olsson, L., Eklundhb, L., Ardo, J., November 2005. A recent greening of the Sahel-trends, patterns and potential causes. Journal of Arid Environments $63(3), 556-566$.

Oppenheim, A., Schafer, R., Buck, J., 1999. Discrete-Time Signal Processing, 2nd Edition. Prentice-Hall Signal Processing series, New Jersey, USA.

Paola, J. D., Schowengerdt, R. A., July 1995. A detailed comparison of backpropagation neural network and maximum likelihood classifiers for urban land use classification. IEEE Transactions on Geosience and Remote Sensing 33 (4), 981-996.

Roy, D., Jin, Y., Lewis, P., Justice, C., July 2005. Prototyping a global algorithm for systematic fire-affected area mapping using MODIS time series data. Remote Sensing of Environment 97 (2), 137-162.

Salmon, B., et al., June 2011. Unsupervised land cover change detection: Meaningful sequential time series analysis. IEEE Journal of Selected Topics in Applied Earth Observations and Remote Sensing 4 (2), 327-335.

Schaaf, C., et al., November 2002. First operational BRDF, albedo nadir reflectance products from MODIS. Journal of Remote Sensing of Environment 83 (1-2), 135-148. 
Townshend, J. R. G., Justice, C. O., February 1988. Selecting the spatial resolution of satellite sensors required for global monitoring of land transformations. International Journal of Remote Sensing 9 (2), 187-236.

Vanacker, V., Linderman, M., Lupo, F., Flasse, S., Lambin, E., January 2005. Impact of short-term rainfall fluctuation on inter-annual land cover change in sub-Saharan Africa. Global Ecology and Biogeography 14 (2), 123-135.

Vermaak, J., Botha, E. C., February 1998. Recurrent neural networks for short-term load forecasting. IEEE Transactions on Power Systems 13 (1), $126-132$.

Wang, X., Xiu-Xia, L., Sun, J., August 18-21, 2005. A new approach of neural networks to time-varying database classification. In: Proceedings Machine Learning and Cybernetics 2005. Vol. 4. Guangzhou, China, pp. 2050-2054.

Wanner, W., et al., July 1997. Global retrieval of bidirectional reflectance and Albedo over land from EOS MODIS and MISR data: theory and algorithm. Journal of Geophyshical Research 102 (D14), 17143-17162.

Westra, T., Wulf, R. R. D., April 2007. Monitoring Sahelian floodplains using Fourier analysis of MODIS time-series data and artificial neural networks. International Journal of Remote Sensing 28 (7), 1595-1610. 


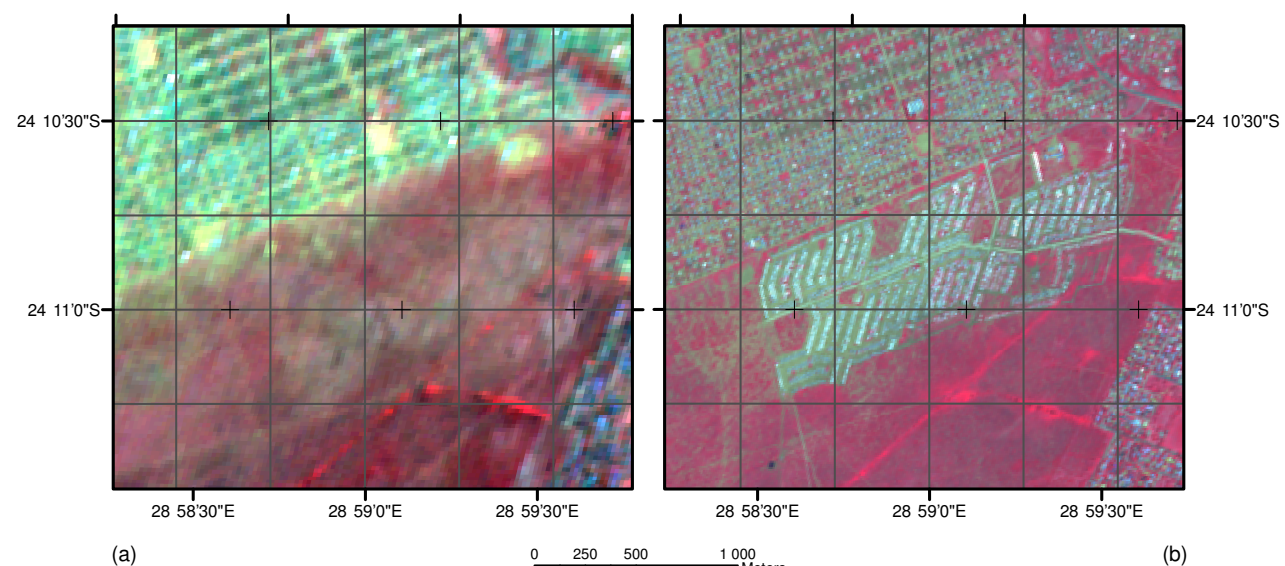

Figure 1: A land cover change of natural vegetation to human settlement in Sekuruwe. The Sekuruwe human settlement is located in the Limpopo province, South Africa. The SPOT2 image (RGB 321) was acquired on 2 May 2000 of the natural vegetation area (a) and a SPOT5 (RGB 321) image was acquired on 1 May 2007 of a newly developed human settlement (b). The SPOT2 and SPOT5 image is projected to a MODIS sinusoidal WGS84 projection and is overlay with a MODIS 500-meter coordinate grid.

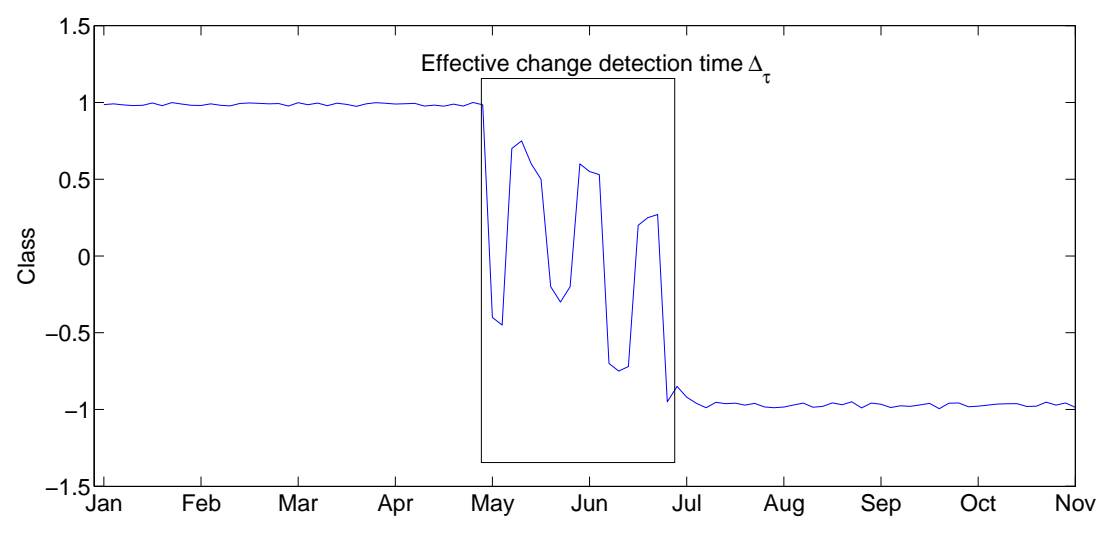

Figure 2: An illustrative example of the effective change detection time $\Delta_{\tau}$, which is defined as the region of uncertainty in the classifier output when land cover change occurs within the MODIS time series. 


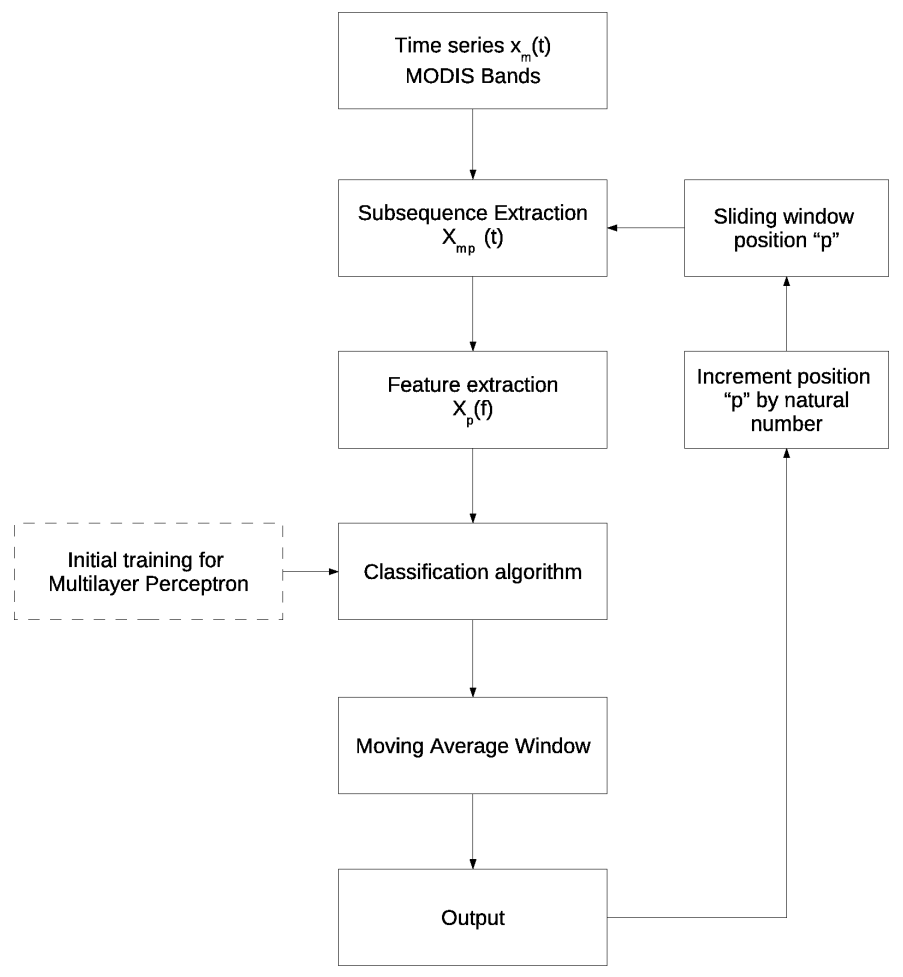

Figure 3: Feature extraction from the MODIS spectral bands that are processed for the MLP to detect land cover change. 


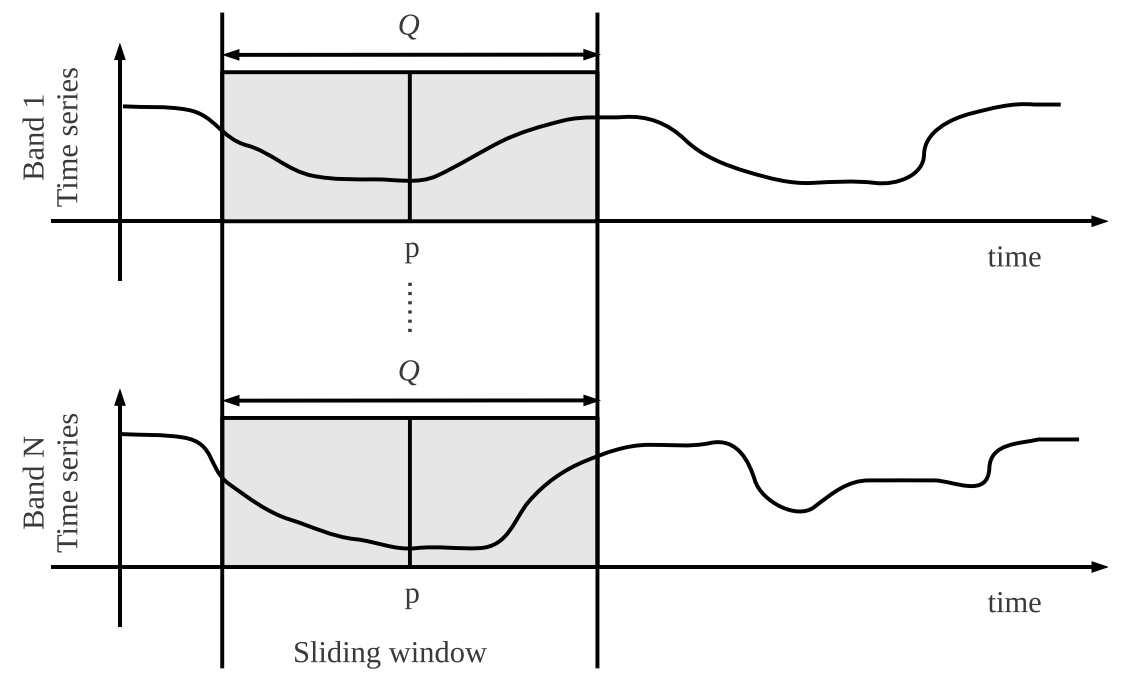

Figure 4: Temporal sliding window used to define a subsequence of the time series for classification and change detection.

Table 1: Number of pixels per land cover type, per study area used for training, validation and testing data sets.

\begin{tabular}{|c|r|r|r|}
\hline Study Area & $\begin{array}{c}\text { Training } \\
\text { Time Series }\end{array}$ & $\begin{array}{c}\text { Validation } \\
\text { Time Series }\end{array}$ & $\begin{array}{c}\text { Simulated Change } \\
\text { Time Series }\end{array}$ \\
\hline Area 1 - Vegetation & 225 & 225 & 84 \\
Area 1 - Settlement & 82 & 82 & 84 \\
Area 2 - Vegetation & 42 & 43 & 42 \\
Area 2 - Settlement & 162 & 162 & 42 \\
Area 3 - Vegetation & 56 & 57 & 56 \\
Area 3 - Settlement & 77 & 77 & 56 \\
\hline Total - Vegetation & 323 & 325 & 182 \\
Total - Settlement & 321 & 321 & 182 \\
\hline
\end{tabular}



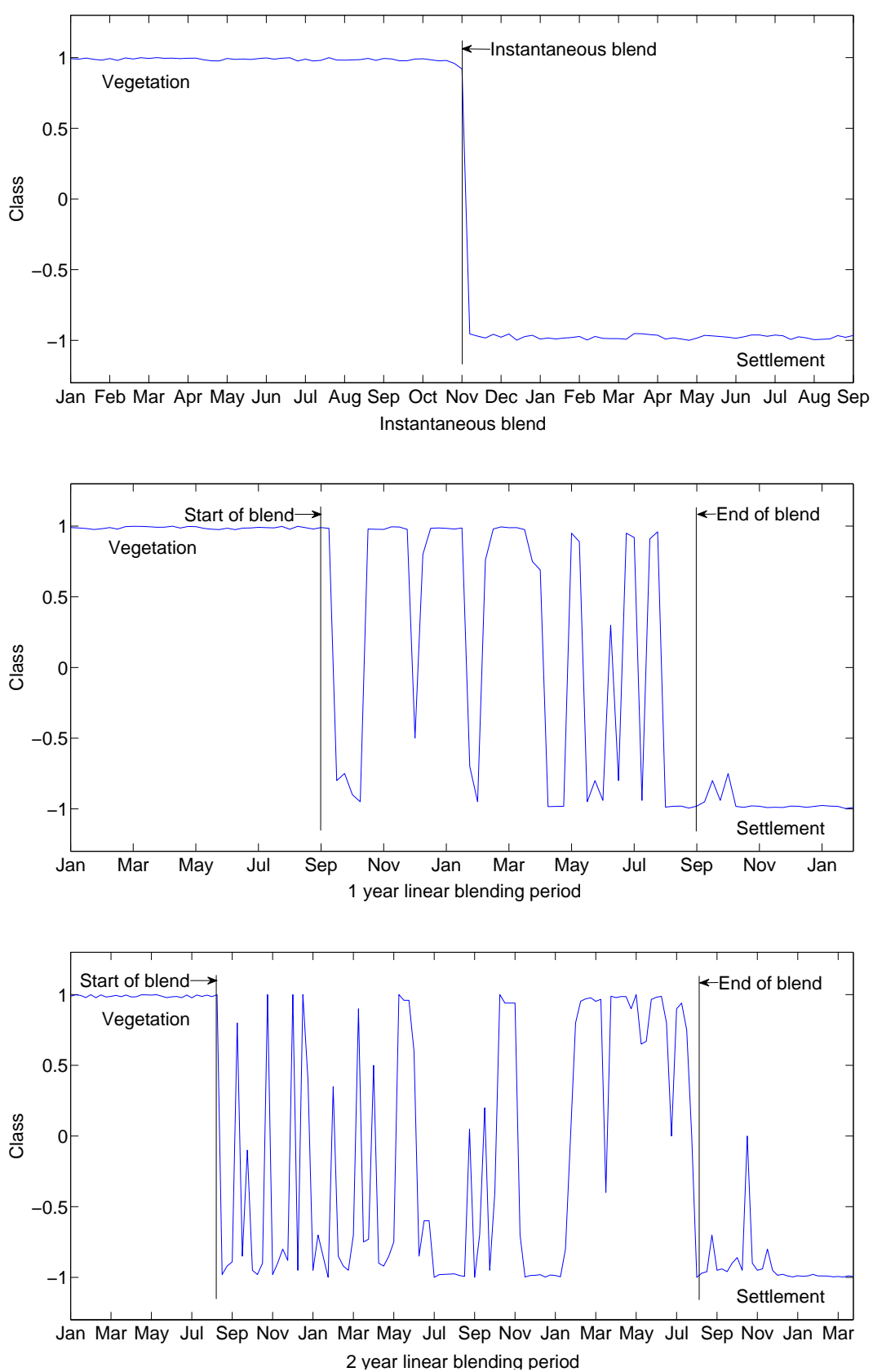

Figure 5: MLP classification for simulated land cover change from natural vegetation to settlement. The top inset is for instantaneous simulated land cover change, the middle inset is for a land cover change over 12 month blending period and the bottom inset is for a land cover change over 24 months blending period. 
Table 2: Classification accuracy of the batch mode MLP on the validation set. Each entry gives the average classification accuracy for each study area, calculated over 15 repeated independent experiments (percentage coefficient of variation shown in parentheses). The average classification accuracy is given in percentage for each of the classes over different temporal sliding window lengths (6,12 and 18 months) and number of spectral bands used (NDVI, 2 spectral bands and all 7 spectral bands).

\begin{tabular}{|c|c|c|c|c|c|c|c|c|c|}
\hline Area & \multicolumn{9}{|c|}{ Study Area 1 : Koelemansrus (28.85E, 24.23S) } \\
\hline Input vector $X_{p}(f)$ & & NDVI & & & 2 bands & & & 7 bands & \\
\hline Sliding window length & 6 months & 12 months & 18 months & 6 months & 12 months & 18 months & 6 months & 12 months & 18 months \\
\hline Vegetation validation & $\begin{array}{r}93.2 \\
(11.1)\end{array}$ & $\begin{array}{r}91.3 \\
(12.9)\end{array}$ & $\begin{array}{r}89.9 \\
(15.5)\end{array}$ & $\begin{array}{r}94.4 \\
(8.6)\end{array}$ & $\begin{array}{r}96.0 \\
(3.9)\end{array}$ & $\begin{array}{r}95.9 \\
(3.5)\end{array}$ & $\begin{array}{r}96.9 \\
(4.6)\end{array}$ & $\begin{array}{r}98.6 \\
(1.5)\end{array}$ & $\begin{array}{r}99.0 \\
(1.2)\end{array}$ \\
\hline Settlement validation & $\begin{array}{r}27.7 \\
(98.2)\end{array}$ & $\begin{array}{r}56.7 \\
(46.6)\end{array}$ & $\begin{array}{r}62.1 \\
(37.0)\end{array}$ & $\begin{array}{r}70.8 \\
(30.6)\end{array}$ & $\begin{array}{r}83.0 \\
(13.0)\end{array}$ & $\begin{array}{r}83.9 \\
(11.9)\end{array}$ & $\begin{array}{r}78.5 \\
(28.7)\end{array}$ & $\begin{array}{l}89.7 \\
(9.0)\end{array}$ & $\begin{array}{l}90.3 \\
(7.8)\end{array}$ \\
\hline Overall validation & 75.7 & 82.1 & 82.5 & 88.1 & 92.6 & 92.6 & 92.0 & 96.2 & 96.7 \\
\hline
\end{tabular}

\begin{tabular}{|c|c|c|c|c|c|c|c|c|c|}
\hline Area & \multicolumn{9}{|c|}{ Study Area 2 : Seshego $(29.40 \mathrm{E}, 23.81 \mathrm{~S})$} \\
\hline Input vector $X_{p}(f)$ & & NDVI & & & 2 bands & & & 7 bands & \\
\hline Sliding window length & 6 months & 12 months & 18 months & 6 months & 12 months & 18 months & 6 months & 12 months & 18 months \\
\hline Vegetation validation & $\begin{array}{r}44.9 \\
(83.1)\end{array}$ & $\begin{array}{r}70.8 \\
(42.8)\end{array}$ & $\begin{array}{r}77.3 \\
(34.5)\end{array}$ & $\begin{array}{r}61.9 \\
(37.8)\end{array}$ & $\begin{array}{r}78.0 \\
(15.9)\end{array}$ & $\begin{array}{r}81.7 \\
(12.7)\end{array}$ & $\begin{array}{r}90.8 \\
(5.6)\end{array}$ & $\begin{array}{r}95.0 \\
(26)\end{array}$ & $\begin{array}{r}95.7 \\
(23)\end{array}$ \\
\hline Settlement validation & $\begin{array}{r}95.8 \\
(5.9) \\
\end{array}$ & $\begin{array}{r}94.7 \\
(3.8) \\
\end{array}$ & $\begin{array}{r}95.0 \\
(2.6) \\
\end{array}$ & $\begin{array}{r}96.2 \\
(4.2) \\
\end{array}$ & $\begin{array}{r}98.0 \\
(2.3) \\
\end{array}$ & $\begin{array}{r}98.3 \\
(1.8) \\
\end{array}$ & $\begin{array}{r}98.1 \\
(2.7) \\
\end{array}$ & $\begin{array}{r}98.9 \\
(0.9) \\
\end{array}$ & $\begin{array}{r}98.8 \\
(0.7) \\
\end{array}$ \\
\hline Overall validation & 85.1 & 89.7 & 91.3 & 89.0 & 93.8 & 94.9 & 96.6 & 98.1 & 98.1 \\
\hline
\end{tabular}

\begin{tabular}{|c|c|c|c|c|c|c|c|c|c|}
\hline Area & \multicolumn{9}{|c|}{ Study Area 3 : Sekuruwe (28.94E, 23.94S) } \\
\hline Input vector $X_{p}(f)$ & & NDVI & & & 2 bands & & & 7 bands & \\
\hline Sliding window length & 6 months & 12 months & 18 months & 6 months & 12 months & 18 months & 6 months & 12 months & 18 months \\
\hline Vegetation validation & $\begin{array}{r}45.0 \\
(70.7)\end{array}$ & $\begin{array}{r}55.8 \\
(45.5)\end{array}$ & $\begin{array}{r}60.7 \\
(38.4)\end{array}$ & $\begin{array}{r}63.6 \\
(30.2)\end{array}$ & $\begin{array}{r}67.5 \\
(24.4)\end{array}$ & $\begin{array}{r}69.9 \\
(20.9)\end{array}$ & $\begin{array}{r}87.4 \\
(9.0)\end{array}$ & $\begin{array}{r}91.4 \\
(4.2)\end{array}$ & $\begin{array}{r}91.5 \\
(3.7)\end{array}$ \\
\hline Settlement validation & $\begin{array}{r}82.7 \\
(20.2)\end{array}$ & $\begin{array}{r}82.0 \\
(16.5)\end{array}$ & $\begin{array}{r}80.1 \\
(15.7)\end{array}$ & $\begin{array}{r}82.0 \\
(14.9)\end{array}$ & $\begin{array}{r}85.4 \\
(8.8)\end{array}$ & $\begin{array}{r}88.8 \\
(6.9)\end{array}$ & $\begin{array}{r}87.8 \\
(11.0)\end{array}$ & $\begin{array}{r}93.0 \\
(4.3)\end{array}$ & $\begin{array}{r}94.6 \\
(3.4)\end{array}$ \\
\hline Overall validation & 66.7 & 71.3 & 71.8 & 74.2 & 77.8 & 80.8 & 87.6 & 92.3 & 93.3 \\
\hline
\end{tabular}


Table 3: Classification accuracy of the iteratively retrained MLP on the validation set. Each entry gives the average classification accuracy for each study area, calculated over 15 repeated independent experiments (percentage coefficient of variation shown in parentheses). The average classification accuracy is given in percentage for each of the classes over different temporal sliding window lengths (6, 12 and 18 months) and number of spectral bands used (NDVI, 2 spectral bands and all 7 spectral bands).

\begin{tabular}{|c|c|c|c|c|c|c|c|c|c|}
\hline Area & \multicolumn{9}{|c|}{ Study Area 1 : Koelemansrus (28.85E, 24.23S) } \\
\hline Input vector $X_{p}(f)$ & & NDVI & & & 2 bands & & & 7 bands & \\
\hline Sliding window length & 6 months & 12 months & 18 months & 6 months & 12 months & 18 months & 6 months & 12 months & 18 months \\
\hline Vegetation validation & $\begin{array}{r}91.4 \\
(5.9)\end{array}$ & $\begin{array}{r}93.4 \\
(3.4)\end{array}$ & $\begin{array}{r}94.4 \\
(2.8)\end{array}$ & $\begin{array}{r}95.9 \\
(2.6)\end{array}$ & $\begin{array}{r}96.7 \\
(2.1)\end{array}$ & $\begin{array}{r}97.2 \\
(1.7)\end{array}$ & $\begin{array}{r}97.7 \\
(1.5)\end{array}$ & $\begin{array}{r}98.3 \\
(1.0)\end{array}$ & $\begin{array}{r}98.5 \\
(0.9)\end{array}$ \\
\hline Settlement validation & $\begin{array}{r}70.8 \\
(22.0)\end{array}$ & $\begin{array}{r}76.9 \\
(9.1)\end{array}$ & $\begin{array}{r}77.3 \\
(8.5)\end{array}$ & $\begin{array}{r}83.0 \\
(7.6)\end{array}$ & $\begin{array}{r}85.9 \\
(5.9)\end{array}$ & $\begin{array}{r}87.9 \\
(4.7)\end{array}$ & $\begin{array}{c}91.4 \\
(4.1)\end{array}$ & $\begin{array}{r}92.8 \\
(3.0)\end{array}$ & $\begin{array}{r}92.8 \\
(3.1)\end{array}$ \\
\hline Overall validation & 85.9 & 89.0 & 89.8 & 92.4 & 93.8 & 94.7 & 96.0 & 96.8 & 97.0 \\
\hline
\end{tabular}

\begin{tabular}{|c|c|c|c|c|c|c|c|c|c|}
\hline Area & \multicolumn{9}{|c|}{ Study Area $2:$ Seshego $(29.40 \mathrm{E}, 23.81 \mathrm{~S})$} \\
\hline Input vector $X_{p}(f)$ & & NDVI & & & 2 bands & & & 7 bands & \\
\hline Sliding window length & 6 months & 12 months & 18 months & 6 months & 12 months & 18 months & 6 months & 12 months & 18 months \\
\hline Vegetation validation & $\begin{array}{r}70.6 \\
(28.1)\end{array}$ & $\begin{array}{r}81.2 \\
(11.1)\end{array}$ & $\begin{array}{r}82.4 \\
(10.7)\end{array}$ & $\begin{array}{r}74.4 \\
(16.3)\end{array}$ & $\begin{array}{r}84.2 \\
(9.1)\end{array}$ & $\begin{array}{r}85.0 \\
(8.8)\end{array}$ & $\begin{array}{r}91.5 \\
(4.7)\end{array}$ & $\begin{array}{r}93.2 \\
(3.5)\end{array}$ & $\begin{array}{r}93.8 \\
(3.4)\end{array}$ \\
\hline Settlement validation & $\begin{array}{r}93.6 \\
(4.6) \\
\end{array}$ & $\begin{array}{r}94.7 \\
(2.5)\end{array}$ & $\begin{array}{r}94.9 \\
(2.3) \\
\end{array}$ & $\begin{array}{r}96.4 \\
(2.6)\end{array}$ & $\begin{array}{r}97.6 \\
(1.7) \\
\end{array}$ & $\begin{array}{r}97.7 \\
(1.6) \\
\end{array}$ & $\begin{array}{r}98.3 \\
(1.3) \\
\end{array}$ & $\begin{array}{r}98.5 \\
(1.1) \\
\end{array}$ & $\begin{array}{r}98.6 \\
(1.0) \\
\end{array}$ \\
\hline Overall validation & 88.8 & 91.8 & 92.3 & 91.8 & 94.8 & 95.0 & 96.9 & 97.4 & 97.6 \\
\hline
\end{tabular}

\begin{tabular}{|c|c|c|c|c|c|c|c|c|c|}
\hline Area & \multicolumn{9}{|c|}{ Study Area 3 : Sekuruwe $(28.94 \mathrm{E}, 23.94 \mathrm{~S})$} \\
\hline Input vector $X_{p}(f)$ & & NDVI & & & 2 bands & & & 7 bands & \\
\hline Sliding window length & 6 months & 12 months & 18 months & 6 months & 12 months & 18 months & 6 months & 12 months & 18 months \\
\hline Vegetation validation & $\begin{array}{r}49.1 \\
(61.0)\end{array}$ & $\begin{array}{r}66.3 \\
(24.2)\end{array}$ & $\begin{array}{r}68.0 \\
(20.22\end{array}$ & $\begin{array}{r}67.6 \\
(22.3)\end{array}$ & $\begin{array}{r}75.5 \\
(14.1)\end{array}$ & $\begin{array}{r}79.9 \\
(11.0)\end{array}$ & $\begin{array}{r}85.9 \\
(7.7)\end{array}$ & $\begin{array}{r}88.5 \\
(6.1)\end{array}$ & $\begin{array}{r}88.8 \\
(5.8)\end{array}$ \\
\hline Settlement validation & $\begin{array}{r}80.7 \\
(22.7)\end{array}$ & $\begin{array}{r}76.1 \\
(15.9)\end{array}$ & $\begin{array}{r}77.8 \\
(13.2)\end{array}$ & $\begin{array}{r}80.8 \\
(11.7)\end{array}$ & $\begin{array}{r}82.9 \\
(7.3)\end{array}$ & $\begin{array}{r}84.3 \\
(7.2)\end{array}$ & $\begin{array}{r}90.2 \\
(5.2)\end{array}$ & $\begin{array}{r}91.7 \\
(4.0)\end{array}$ & $\begin{array}{r}91.6 \\
(4.0)\end{array}$ \\
\hline Overall validation & 67.3 & 71.9 & 73.6 & 75.2 & 79.8 & 82.4 & 88.4 & 90.3 & 90.4 \\
\hline
\end{tabular}


Table 4: The land cover change detection accuracy for all the study areas was evaluated using a batch mode trained MLP on the simulated land cover change set. In this table the input vectors are compared to the different combinations of sliding window lengths $Q$.

\begin{tabular}{|l|rrr|rrr|rrr|}
\hline Area & \multicolumn{7}{|c|}{ Study Area 1 : Koelemansrus (28.85E, 24.23S) } \\
\hline Input vector $X_{p}(f)$ & \multicolumn{7}{|c|}{ NDVI } & \multicolumn{7}{c|}{ bands } & 7 bands \\
Sliding window length & 6 months & 12 months & 18 months & 6 months & 12 months & 18 months & 6 months & 12 months & 18 months \\
\hline True positive & 4.5 & 55.3 & 60.4 & 72.5 & 81.9 & 83.8 & 79.3 & 90.1 & 91.5 \\
True negative & 95.6 & 73.9 & 75.5 & 79.8 & 85.3 & 87.3 & 82.3 & 91.2 \\
False positive & 4.4 & 26.1 & 24.5 & 20.2 & 14.7 & 12.7 & 17.7 & 8.9 & 7.7 \\
False negative & 95.5 & 44.7 & 39.6 & 27.5 & 18.1 & 16.2 & 20.7 & 9.9 \\
\hline
\end{tabular}

\begin{tabular}{|c|c|c|c|c|c|c|c|c|c|}
\hline Area & & & & tudy Area & : Seshego & $.40 \mathrm{E}, 23.81$ & & & \\
\hline Input vector $X_{p}(f)$ & & NDVI & & & 2 bands & & & 7 bands & \\
\hline Sliding window length & 6 months & 12 months & 18 months & 6 months & 12 months & 18 months & 6 months & 12 months & 18 months \\
\hline True positive & 52.4 & 81.1 & 83.4 & 73.3 & 84.7 & 85.3 & 90.3 & 94.6 & 94.7 \\
\hline True negative & 70.5 & 81.8 & 83.7 & 77.8 & 84.2 & 86.0 & 90.9 & 95.1 & 95.7 \\
\hline False positive & 29.5 & 18.2 & 16.3 & 22.2 & 15.8 & 14.0 & 9.1 & 4.9 & 4.3 \\
\hline False negative & 47.6 & 18.9 & 16.6 & 26.7 & 15.3 & 14.7 & 9.7 & 5.4 & 5.3 \\
\hline
\end{tabular}

\begin{tabular}{|l|rrr|rrr|rrr|}
\hline Area & \multicolumn{7}{|c|}{ Study Area 3 : Sekuruwe (28.94E, 23.94S) } \\
\hline Input vector $X_{p}(f)$ & \multicolumn{3}{|c|}{ NDVI } & \multicolumn{7}{c|}{$\begin{array}{c}\text { bands } \\
\text { Sliding window length }\end{array}$} & 6 months & 12 months & 18 months & 6 months & 12 months & 18 months & 6 months & 12 bands \\
\hline True positive & 49.2 & 51.2 & 52.2 & 58.9 & 67.7 & 74.5 & 84.8 & 88.0 & 18 months \\
True negative & 65.7 & 65.5 & 64.2 & 69.9 & 75.1 & 77.8 & 85.0 & 88.5 & 89.0 \\
False positive & 34.3 & 34.5 & 35.8 & 30.1 & 24.9 & 22.2 & 15.0 & 11.5 & 11.0 \\
False negative & 50.8 & 48.8 & 47.8 & 41.1 & 32.3 & 25.5 & 15.2 & 12.0 & 12.0 \\
\hline
\end{tabular}


Table 5: The land cover change detection accuracy for all the study areas was evaluated using an iteratively retrained mode MLP on the simulated land cover change set. In this table the input vectors are compared to the different combinations of sliding window lengths $Q$.

\begin{tabular}{|l|rrr|rrr|rrr|}
\hline Area & \multicolumn{7}{|c|}{ Study Area 1 : Koelemansrus (28.85E, 24.23S) } \\
\hline Input vector $X_{p}(f)$ & \multicolumn{3}{|c|}{ NDVI } & \multicolumn{7}{c|}{ bands } & \multicolumn{3}{c|}{ bands } \\
Sliding window length & 6 months & 12 months & 18 months & 6 months & 12 months & 18 months & 6 months & 12 months & 18 months \\
\hline True positive & 75.6 & 77.5 & 78.1 & 86.4 & 90.5 & 91.8 & 94.1 & 94.3 \\
True negative & 79.4 & 80.8 & 80.9 & 88.3 & 91.4 & 91.4 & 94.2 & 94.5 \\
False positive & 20.6 & 19.3 & 19.1 & 11.7 & 8.6 & 8.6 & 5.8 & 5.5 \\
False negative & 24.4 & 22.5 & 21.9 & 13.6 & 9.5 & 8.2 & 5.9 & 5.7 \\
\hline
\end{tabular}

\begin{tabular}{|c|c|c|c|c|c|c|c|c|c|}
\hline Area & \multicolumn{9}{|c|}{ Study Area 2: Seshego $(29.40 \mathrm{E}, 23.81 \mathrm{~S})$} \\
\hline Input vector $X_{p}(f)$ & \multirow{2}{*}{\multicolumn{3}{|c|}{$\begin{array}{c}\text { NDVI } \\
12 \text { months }\end{array}$}} & \multicolumn{3}{|c|}{2 bands } & \multicolumn{3}{|c|}{7 bands } \\
\hline Sliding window length & & & 18 months & 6 months & 12 months & 18 months & 6 months & 12 months & 18 months \\
\hline True positive & 79.4 & 81.7 & 84.1 & 82.5 & 88.3 & 89.5 & 92.5 & 92.5 & 92.6 \\
\hline True negative & 80.6 & 82.7 & 83.3 & 84.4 & 87.7 & 88.5 & 92.2 & 92.2 & 92.7 \\
\hline False positive & 19.4 & 17.3 & 16.7 & 15.6 & 12.3 & 11.5 & 7.8 & 7.8 & 7.3 \\
\hline False negative & 20.6 & 18.3 & 15.9 & 17.5 & 11.7 & 10.5 & 7.5 & 7.5 & 7.4 \\
\hline Area & \multicolumn{9}{|c|}{ Study Area 3 : Sekuruwe (28.94E, 23.94S) } \\
\hline Input vector $X_{p}(f)$ & & NDVI & & & 2 bands & & & 7 bands & \\
\hline Sliding window length & 6 months & 12 months & 18 months & 6 months & 12 months & 18 months & 6 months & 12 months & 18 months \\
\hline True positive & 58.4 & 64.3 & 69.0 & 67.9 & 76.4 & 79.6 & 89.6 & 89.9 & 90.2 \\
\hline True negative & 67.0 & 68.5 & 71.1 & 72.4 & 80.0 & 80.0 & 89.9 & 91.3 & 91.4 \\
\hline False positive & 33.0 & 31.5 & 28.9 & 27.6 & 20.0 & 20.0 & 10.1 & 8.7 & 8.6 \\
\hline False negative & 41.6 & 35.7 & 31.0 & 32.1 & 23.6 & 20.4 & 10.4 & 10.1 & 9.8 \\
\hline
\end{tabular}


Table 6: The land cover change detection accuracy for real land cover change in the Sekuruwe study area. In this table the different input vectors (NDVI, 2 spectral bands and all 7 spectral bands) are compared to the length of the sliding window $Q$ (6, 12 and 18 months). Each entry in both tables gives the change detection accuracy for the Sekuruwe study area, calculated over 15 repeated independent experiments.

\begin{tabular}{|l|rrr|rrr|rr|}
\hline Area & \multicolumn{7}{|c|}{ Study Area 3 : Sekuruwe (28.94E, 23.94S) } \\
\hline $\begin{array}{l}\text { Input vector } X_{p}(f) \\
\text { Sliding window length }\end{array}$ & 6 months & $\begin{array}{c}\text { NDVI } \\
12 \text { months }\end{array}$ & 18 months & 6 months & $\begin{array}{c}2 \text { bands } \\
12 \text { months }\end{array}$ & 18 months & 6 months & 7 bands \\
12 months & 18 months \\
\hline $\begin{array}{l}\text { Batch mode MLP } \\
\text { Detection accuracy }\end{array}$ & 51.7 & 54.0 & 54.1 & 56.6 & 61.9 & 64.0 & 82.6 & 84.8 \\
\hline $\begin{array}{l}\text { Iterative retrained MLP } \\
\text { Detection accuracy }\end{array}$ & 57.8 & 62.9 & 65.7 & 62.4 & 68.8 & 71.6 & 83.1 & 86.6 \\
\hline
\end{tabular}


Table 7: Effective change detection time $\Delta_{\tau}$ for simulated land cover change using a batch mode trained MLP for each of the temporal sliding window lengths and input vectors. Each entry gives the average number of days for each study area, calculated over 15 repeated independent experiments. The sliding window has a length $\mathrm{Q}$ of 6,12 , and, 18 months which denotes 23, 46, and, 69 MODIS acquisitions respectively at a fixed rate of 8-day increments within a 16 day composite period.

\begin{tabular}{|l|r|r|r|}
\hline Area & \multicolumn{3}{|c|}{ Study Area 1 } \\
\hline Sliding window & 6 months & 12 months & 18 months \\
\hline NDVI & - & 127 & 189 \\
2 Bands & 93 & 128 & 170 \\
7 Bands & 90 & 102 & 163 \\
\hline \hline Area & \multicolumn{3}{|c|}{ Study Area 2 } \\
\hline Sliding window & 6 months & 12 months & 18 months \\
\hline NDVI & 286 & 288 & 378 \\
2 Bands & 85 & 269 & 287 \\
7 Bands & 79 & 251 & 264 \\
\hline \hline Area & \multicolumn{3}{|c|}{ Study Area 3 } \\
\hline Sliding window & 6 months & 12 months & 18 months \\
\hline NDVI & 203 & 309 & 409 \\
2 Bands & 208 & 289 & 353 \\
7 Bands & 91 & 158 & 256 \\
\hline
\end{tabular}

Table 8: Effective change detection time $\Delta_{\tau}$ for simulated land cover change using an iteratively retrained MLP for each of the temporal sliding window lengths and input vectors. Each entry gives the average number of days for each study area, calculated over 15 repeated independent experiments. The sliding window has a length $\mathrm{Q}$ of 6,12 , and, 18 months which denotes 23, 46, and, 69 MODIS acquisitions respectively at a fixed rate of 8-day increments within a 16 day composite period.

\begin{tabular}{|l|r|r|r|}
\hline Area & \multicolumn{3}{|c|}{ Study Area 1 } \\
\hline Sliding window & 6 months & 12 months & 18 months \\
\hline NDVI & 136 & 185 & 196 \\
2 Bands & 76 & 109 & 158 \\
7 Bands & 70 & 73 & 111 \\
\hline \hline Area & \multicolumn{3}{|c|}{ Study Area 2 } \\
\hline Sliding window & 6 months & 12 months & 18 months \\
\hline NDVI & 97 & 158 & 232 \\
2 Bands & 99 & 123 & 221 \\
7 Bands & 81 & 116 & 214 \\
\hline \hline Area & \multicolumn{3}{|c|}{ Study Area 3 } \\
\hline Sliding window & 6 months & 12 months & 18 months \\
\hline NDVI & 115 & 208 & 271 \\
2 Bands & 105 & 195 & 264 \\
7 Bands & 67 & 179 & 241 \\
\hline
\end{tabular}


Table 9: The average computational time for both the batch mode MLP and iteratively retrained MLP is given for different temporal sliding window lengths (6, 12 and 18 months) and number of spectral bands used (NDVI, 2 spectral bands and all 7 spectral bands) in the Sekuruwe study area. Each entry gives the average computational time calculated over 15 repeated independent experiments. The fastest computational time is used as based comparison with the other entries which are fractional increases of this base.

\begin{tabular}{|c|c|c|c|}
\hline Input vector $X_{p}(f)$ & \multicolumn{3}{|c|}{ NDVI } \\
\hline Sliding window & 6 months & 12 months & 18 months \\
\hline Number of input nodes & 5 & 5 & 5 \\
\hline Number of hidden nodes & 5 & 8 & 13 \\
\hline Computing time - Batch mode MLP & 1.06 & 1.07 & 1.08 \\
\hline Computing time - Iteratively retrained MLP & 1.00 & 1.03 & 1.04 \\
\hline Input vector $X_{p}(f)$ & \multicolumn{3}{|c|}{2 Spectral Bands } \\
\hline Sliding window & 6 months & 12 months & 18 months \\
\hline Number of input nodes & 10 & 10 & 10 \\
\hline Number of hidden nodes & 8 & 12 & 16 \\
\hline Computing time - Batch mode MLP & 1.17 & 1.21 & 1.22 \\
\hline Computing time - Iteratively retrained MLP & 1.09 & 1.14 & 1.18 \\
\hline Input vector $X_{p}(f)$ & \multicolumn{3}{|c|}{7 Spectral Bands } \\
\hline Sliding window & 6 months & 12 months & 18 months \\
\hline Number of input nodes & 35 & 35 & 35 \\
\hline Number of hidden nodes & 10 & 14 & 19 \\
\hline Computing time - Batch mode MLP & 1.70 & 1.80 & 1.88 \\
\hline Computing time - Iteratively retrained MLP & 1.67 & 1.73 & 1.79 \\
\hline
\end{tabular}

